\title{
Temperature- and quantum phonon effects on Holstein-Hubbard bipolarons
}

\author{
Martin Hohenadlel* and Wolfgang von der Linden \\ Institute for Theoretical and Computational Physics, \\ Graz University of Technology, Petersgasse 16, 8010 Graz, Austria
}

\begin{abstract}
The one-dimensional Holstein-Hubbard model with two electrons of opposite spin is studied using an extension of a recently developed quantum Monte Carlo method, and a very simple yet rewarding variational approach, both based on a canonically transformed Hamiltonian. The quantum Monte Carlo method yields very accurate results in the regime of small but finite phonon frequencies, characteristic of many strongly correlated materials such as, e.g., the cuprates and the manganites. The influence of electron-electron repulsion, phonon frequency and temperature on the bipolaron state is investigated. Thermal dissociation of the intersite bipolaron is observed at high temperatures, and its relation to an existing theory of the manganites is discussed.
\end{abstract}

PACS numbers: 63.20.Kr, 71.27.+a, 71.38.-k, 71.38.Mx 02.70.Ss

\section{INTRODUCTION}

In recent years, the formation and properties of bipolarons, consisting of two electrons forming a pair in real space, have received considerable interest due to their potential role, e.g., in high-temperature superconductivity. Theories based on bipolaron formation have been proposed for the superconducting transition in the cuprates, $\frac{1}{,}$ and the metal-insulator transition and colossal magnetoresistance in the manganites ${ }^{2.3}$ Despite some fundamental problems, $\stackrel{4,5,6}{,}$ they are still issue of ongoing discussion.

Many interesting materials fall into the adiabatic regime of small but finite phonon frequencies and intermediate to strong electron-phonon coupling. For such parameters, analytical approaches based on, e.g., perturbation theory, do not give reliable results. In contrast, computational methods represent a very powerful instrument to obtain exact, unbiased information, and a lot of numerical work has recently been devoted to an understanding of the Holstein and the Holstein-Hubbard ( $\mathrm{HH})$ model.

In this paper, we present a simple but surprisingly accurate variational approach to the $\mathrm{HH}$ bipolaron. More importantly, we extend a recently developed quantum Monte Carlo (QMC) $\operatorname{method}^{7}$ to the case of two electrons of opposite spin. The resulting algorithm is used to study bipolaron formation in the one-dimensional $\mathrm{HH}$ model, focusing on the adiabatic regime. While the groundstate properties of the $\mathrm{HH}$ bipolaron are rather well understood, here we exploit the capability of the QMC approach to also study finite temperatures. We find that, in particular, the weakly bound intersite bipolaron is susceptible to thermal dissociation. Furthermore, in contrast to previous studies, we are able to consider a very large range of the electron-phonon and electron-electron interaction.

The outline of this work is as follows. In Sec. II we discuss the HH model with two electrons, while in Sec. III we present an extended Lang-Firsov transformation with nonlocal lattice displacements. Section IV features the extension of the QMC method to the bipolaron problem, and Sec. $\bar{\nabla}$ covers the variational approach. Results are presented in Sec. VI and Sec. VII contains our conclusions.

\section{THE HOLSTEIN-HUBBARD MODEL}

The $\mathrm{HH}$ model is defined in terms of dimensionless phonon by the Hamiltonian

$$
\begin{aligned}
H= & \underbrace{-t \sum_{\langle i j\rangle \sigma} c_{i \sigma}^{\dagger} c_{j \sigma}}_{K}+\underbrace{\frac{\omega}{2} \sum_{i}\left(\hat{p}_{i}^{2}+\hat{x}_{i}^{2}\right)}_{P=P_{p}+P_{x}} \\
& \underbrace{-\alpha \sum_{i} \hat{n}_{i} \hat{x}_{i}}_{I_{\mathrm{ep}}}+\underbrace{U \sum_{i} \hat{n}_{i \uparrow} \hat{n}_{i \downarrow}}_{I_{\mathrm{ee}}},
\end{aligned}
$$

where $K$ describes the hopping of electrons, $P$ corresponds to the sum of the kinetic $\left(P_{p}\right)$ and elastic $\left(P_{x}\right)$ energy of the phonons, and $I_{\mathrm{ep}}, I_{\mathrm{ee}}$ denote the electronphonon (el-ph) and electron-electron (el-el) interaction terms, respectively. Here $c_{i \sigma}^{\dagger}\left(c_{i \sigma}\right)$ creates (annihilates) an electron of spin $\sigma$ at lattice site $i, \hat{x}_{i}$ and $\hat{p}_{i}$ denote the displacement and momentum of a harmonic oscillator at site $i$, and $\hat{n}_{i}=\sum_{\sigma} \hat{n}_{i \sigma}$ with $\hat{n}_{i \sigma}=c_{i \sigma}^{\dagger} c_{i \sigma}$. The third term, $I_{\mathrm{ep}}$, describes the coupling of dispersionless Einstein phonons to the electron occupation number $\hat{n}_{i}$. For doped cuprates or manganites, such a local interaction is expected to be a reasonable approximation as a result of screening. In the first term, the symbol $\langle i j\rangle$ denotes a summation over all nearest-neighbor hopping pairs $(i, j)$ and $(j, i)$. The parameters of the model are the hopping integral $t$, the phonon energy $\omega(\hbar=1)$, the el-ph coupling constant $\alpha$, and the Coulomb repulsion $U>0$. For $U=0$, Eq. (11) is identical to the Holstein model ${ }^{8}$ As in previous work, we introduce the dimensionless coupling constant $\lambda=\alpha^{2} /(\omega W)$, where $W=4 t \mathrm{D}$ is the bare bandwidth in $\mathrm{D}$ dimensions. We further define the parameters $\bar{\omega}=\omega / t$ and $\bar{U}=U / t$, and express all energies in units of $t$. Consequently, the independent parameters 
of the model are $\bar{\omega}, \lambda$, and $\bar{U}$. We shall see below that a very useful quantity is given by the polaron binding energy $E_{\mathrm{P}}=\lambda W / 2$. Finally, throughout this paper, periodic boundary conditions in real space are assumed.

This work is exclusively concerned with the case of two electrons, neglecting the interaction between bipolarons which will definitely be present to some degree in real materials. Furthermore, we restrict our attention to two electrons with opposite spin, i.e., to the singlet bipolaron. A comparison of the singlet and triplet state has recently been given in Ref. 9 .

A review of early work on the bipolaron problem can be found in the book of Alexandrov and Mott 10 Here we focus the discussion on more recent developments. The latter can roughly be divided into two classes depending on the methods employed: (a) variational approaches, 11.12.13.14.15.16.17 and (b) unbiased numerical studies using $\mathrm{ED}, 18.19 .20 .21 .22$ variational diagonalization, $23,24,25,26$ the density-matrix renormalization group (DMRG) $\stackrel{27}{2}$ and QMC ${ }^{28,29}$ Except for the QMC study of de Raedt and Lagendijk, 28 all work was restricted to the ground state. Moreover, even their QMC results were reported only for a single, low temperature. This motivates our study of temperature effects in Sec. IV

While ED and DMRG studies were obtained on clus-

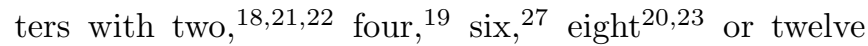
sites,, 24 the variational methods of Bonča et al.$^{25}$ and of Refs. 11 $12,13 \quad 14 \quad 1516 \quad 17$ are only weakly influenced by finite-size effects. An important disadvantage of ED and DMRG is the fact that the phonon Hilbert space has to be truncated, so that these methods can not easily be used to study the adiabatic $(\bar{\omega} \ll 1)$ and/or strongcoupling $(\lambda \gg 1)$ regime. In contrast, no such limitations are imposed on QMC and most variational methods.

Although de Raedt and Lagendijk only considered the adiabatic limit $\bar{\omega}=0$, similar to other authors, 11.12 .14 .15 .28 their method can also be applied for finite phonon frequency ${ }^{28}$ Moreover, it may be generalized to include dispersive phonons. Recently, an extended Holstein model with long-range el-ph interaction has been investigated by Bonča and Trugman ${ }^{30}$ De Raedt and Lagendijk also considered long-range Coulomb interaction, while most other authors only took into account the local Hubbard-type interaction given in Eq. (1), except for Zhang et al. ${ }^{27}$ who have omitted this term in their DMRG calculations. Finally, we would like to point out that bipolaron formation in a model with JahnTeller modes - as present in the perovskite manganiteshas been studied by Shawish et al. 26

\section{TRANSFORMED HAMILTONIANS}

The basis of both the variational approach and the QMC method presented below is the unitary transformation $\tilde{H} \equiv \nu H \nu^{\dagger}$ of the Hamiltonian (1), with $\nu=$ $\exp \left(\mathrm{i} \sum_{i j} \gamma_{i j} \hat{n}_{i} \hat{p}_{j}\right)$ (see Ref. 7). The result is

$$
\begin{aligned}
\tilde{H}= & \underbrace{-t \sum_{\langle i j\rangle \sigma} c_{i \sigma}^{\dagger} c_{j \sigma} e^{i \sum_{l}\left(\gamma_{i l}-\gamma_{j l}\right) \hat{p}_{l}}}_{\tilde{K}}+P \\
& +\underbrace{\sum_{i j} \hat{n}_{j} \hat{x}_{i}\left(\omega \gamma_{i j}-\alpha \delta_{i j}\right)}_{\tilde{I}_{\mathrm{ep}}}+\underbrace{\sum_{i j} v_{i j} \hat{n}_{i} \hat{n}_{j}-\frac{U}{2} \sum_{i} \hat{n}_{i}}_{\tilde{I}_{\mathrm{ee}}},
\end{aligned}
$$

with

$$
v_{i j}=\frac{\omega}{2} \sum_{l} \gamma_{l j} \gamma_{l i}-\alpha \gamma_{i j}+\frac{1}{2} \delta_{i j} U
$$

As discussed in Ref. 7, henceforth also referred to as I, the extended transformation $\nu$ takes into account nonlocal lattice displacements, which are essential for a correct description in the regime $\bar{\omega} \lesssim 1$.

Similar to I, for the QMC method, we resort to the standard Lang-Firsov (LF) transformation ${ }^{31}$ with $\nu_{0}=$ $\exp \left(\mathrm{i} \gamma \sum_{i} \hat{n}_{i} \hat{p}_{i}\right)$. Here $\gamma=\sqrt{\lambda W / \omega}$ has been chosen such that the el-ph coupling term $I_{\mathrm{ep}}$ in Eq. (11) cancels. The transformed Hamiltonian then takes the form

$$
\begin{aligned}
\tilde{H}_{0}= & \underbrace{-t \sum_{\langle i j\rangle \sigma} c_{i \sigma}^{\dagger} c_{j \sigma} e^{\mathrm{i} \gamma\left(\hat{p}_{i}-\hat{p}_{j}\right)}}_{\tilde{K}_{0}}+P \\
& +\underbrace{\left(U-2 E_{\mathrm{P}}\right) \sum_{i} \hat{n}_{i \uparrow} \hat{n}_{i \downarrow}}_{\tilde{I}}-2 E_{\mathrm{P}} .
\end{aligned}
$$

Hence, in contrast to the polaron problem,,$\frac{7}{-}$ the el-el interaction term, resulting from the canonical transformation, does not vanish but instead combines with the Hubbard term.

\section{QUANTUM MONTE CARLO}

The derivation of the QMC algorithm for the bipolaron problem is very similar to the one-electron case, 7 and we shall therefore focus on the differences occurring. Moreover, we restrict the discussion to one dimension.

\section{A. Partition function}

We set out to calculate the partition function $\mathcal{Z}=$ $e^{-\beta \tilde{H}_{0}}$, with $\tilde{H}_{0}$ given by Eq. (4). To this end, we first notice that the last term in Hamiltonian (4) is a constant and can therefore be neglected during the QMC simulation. Using the standard Suzuki-Trotter decomposition we obtain 32

$$
e^{-\beta \tilde{H}_{0}} \approx\left(e^{-\Delta \tau \tilde{K}_{0}} e^{-\Delta \tau P_{\mathrm{p}}} e^{-\Delta \tau P_{\mathrm{x}}} e^{-\Delta \tau \tilde{I}}\right)^{L} \equiv \mathcal{U}^{L},
$$


where $\beta=\left(k_{\mathrm{B}} T\right)^{-1}$ and $\Delta \tau=\beta / L$. Inserting $L$ complete sets of phonon momentum eigenstates and splitting up the trace into a bosonic and a fermionic part we find ${ }^{7}$

$$
\mathcal{Z}_{L}=\operatorname{Tr}_{\mathrm{f}} \int \mathrm{d} p_{1} \mathrm{~d} p_{2} \cdots \mathrm{d} p_{L}\left\langle p_{1}|\mathcal{U}| p_{2}\right\rangle \cdots\left\langle p_{L}|\mathcal{U}| p_{1}\right\rangle,
$$

where $\mathrm{d} p_{\tau} \equiv \prod_{i} \mathrm{~d} p_{i, \tau}$, and $\lim _{L \rightarrow \infty} \mathcal{Z}_{L}=\mathcal{Z}{ }^{32}$ Since the phonon contribution to $\mathcal{U}$ is identical to the singleelectron problem, ${ }^{7}$ we can again integrate out the coordinates $\hat{x}$. Upon defining $\mathcal{D} p=\mathrm{d} p_{1} \mathrm{~d} p_{2} \cdots \mathrm{d} p_{L}$ the partition function becomes

$$
\mathcal{Z}_{L}=C \int \mathcal{D} p w_{\mathrm{b}} w_{\mathrm{f}},
$$

with $C=[2 \pi /(\omega \Delta \tau)]^{N L}$,

$$
\begin{gathered}
w_{\mathrm{b}}=e^{-\Delta \tau S_{\mathrm{b}}}, \quad w_{\mathrm{f}}=\operatorname{Tr}_{\mathrm{f}} \Omega \\
\Omega=\prod_{\tau=1}^{L} e^{-\Delta \tau \tilde{K}_{0, \tau}} e^{-\Delta \tau \tilde{I}} .
\end{gathered}
$$

Here $\tilde{K}_{0, \tau}$ is obtained from $\tilde{K}_{0}$ [Eq. (4)] by replacing $\hat{p}_{i}$ $\left(\hat{p}_{j}\right)$ with $p_{i, \tau}\left(p_{j, \tau}\right)$. The bosonic action has the form

$$
S_{\mathrm{b}}=\sum_{i=1}^{N} \boldsymbol{p}_{i}^{\mathrm{T}} A \boldsymbol{p}_{i},
$$

with $\boldsymbol{p}_{i}=\left(p_{i, 1}, \ldots p_{i, L}\right)$ and a tridiagonal $L \times L$ matrix $A$ defined by

$$
A_{l, l}=\frac{\omega}{2}+\frac{1}{\omega \Delta \tau^{2}}, \quad A_{l, l \pm 1}=-\frac{1}{2 \omega \Delta \tau^{2}} .
$$

As pointed out in I, the representation of $S_{\mathrm{b}}$ given in Eq. (9) permits us to introduce the so-called principal component representation discussed below.

To evaluate the fermionic trace we choose the twoelectron basis states

$$
\left\{|l\rangle \equiv|i, j\rangle \equiv c_{i \uparrow}^{\dagger} c_{j \downarrow}^{\dagger}|0\rangle, \quad i, j=1, \ldots, N\right\},
$$

where we have introduced a combined index $l$ running from 1 to $N^{2}$ in one dimension. We begin with the contribution of the kinetic term $\tilde{K}_{0}$ [Eq. (4)]. It follows that the tight-binding hopping matrix, denoted as $\kappa$, has dimension $N^{2} \times N^{2}$. The exponential of the transformed hopping term can be written as ${ }^{\text {? }}$

$$
e^{-\Delta \tau \tilde{K}_{0, \tau}}=D_{\tau} \kappa D_{\tau}^{\dagger}
$$

where

$$
\left(D_{\tau}\right)_{l l^{\prime}}=\delta_{l l^{\prime}}\left(\delta_{n_{i \uparrow}, 1} \delta_{n_{j \downarrow}, 1}+\delta_{n_{i \downarrow}, 1} \delta_{n_{j \uparrow}, 1}\right) e^{\mathrm{i} \gamma\left(p_{i, \tau}+p_{j, \tau}\right)}
$$

is diagonal in the basis (11).

The second contribution to the matrix $\Omega$ in Eq. (8) comes from the effective el-el interaction term $\tilde{I}$ [Eq. (4)] in terms of the diagonal matrix

$$
\left(\mathcal{V}_{\tau}\right)_{l l^{\prime}}=\delta_{l l^{\prime}} e^{\Delta \tau\left(U-2 E_{\mathrm{P}}\right) \delta_{i j}} .
$$

We would like to emphasize that the random variables $\boldsymbol{p}$ merely enter the diagonal matrix $D$, while the $N^{2} \times N^{2}$ matrices $\mathcal{V}_{\tau}$ and $\kappa$ are fixed throughout the entire MC simulation. Thus, in total, we have

$$
\Omega=\prod_{\tau} D_{\tau} \kappa D_{\tau}^{\dagger} \mathcal{V}_{\tau}
$$

and the fermionic trace is calculated as

$$
\operatorname{Tr}_{\mathrm{f}} \Omega=\sum_{i j}\langle i, j|\Omega| i, j\rangle,
$$

which is identical to the sum over the diagonal elements of the matrix $\Omega$ in the basis (11).

\section{B. Observables}

The first observable of interest is the kinetic energy of the electrons defined as

$$
E_{\mathrm{k}}=-t \sum_{\langle i j\rangle \sigma}\left\langle\tilde{c}_{i \sigma}^{\dagger} \tilde{c}_{j \sigma}\right\rangle=-2 t \sum_{\langle i j\rangle}\left\langle c_{i \uparrow}^{\dagger} c_{j \uparrow} e^{\mathrm{i} \gamma\left(\hat{p}_{i}-\hat{p}_{j}\right)}\right\rangle,
$$

where we have exploited spin symmetry. Following the same steps as in the derivation of the partition function we get

$$
\left\langle\tilde{c}_{i \uparrow}^{\dagger} \tilde{c}_{j \uparrow}\right\rangle=\mathcal{Z}_{L}^{-1} \int \mathcal{D} p w_{\mathrm{b}} e^{\mathrm{i} \gamma\left(p_{i, 1}-p_{j, 1}\right)} \operatorname{Tr}_{\mathrm{f}}\left(\Omega c_{i \uparrow}^{\dagger} c_{j \uparrow}\right) .
$$

Writing out explicitly the fermionic trace we obtain

$$
\begin{aligned}
\operatorname{Tr}_{\mathrm{f}}\left(\Omega c_{i \uparrow}^{\dagger} c_{j \uparrow}\right) & =\sum_{i^{\prime} j^{\prime}}\left\langle i^{\prime}, j^{\prime}\left|\Omega c_{i \uparrow}^{\dagger} c_{j \uparrow}\right| i^{\prime}, j^{\prime}\right\rangle \\
& =\sum_{j^{\prime}}\left\langle j, j^{\prime}|\Omega| i, j^{\prime}\right\rangle
\end{aligned}
$$

and the kinetic energy finally becomes

$$
E_{\mathrm{k}}=-2 t \mathcal{Z}_{L}^{-1} \int \mathcal{D} p w_{\mathrm{b}} \sum_{\langle i j\rangle} \sum_{j^{\prime}} e^{\mathrm{i} \gamma\left(p_{i, 1}-p_{j, 1}\right)}\left\langle j, j^{\prime}|\Omega| i, j^{\prime}\right\rangle .
$$

In addition to $E_{\mathrm{k}}$, we shall also consider the correlation function

$$
\rho(\delta)=\sum_{i}\left\langle\hat{n}_{i \uparrow} \hat{n}_{i+\delta \downarrow}\right\rangle \quad, \quad \delta=0,1, \ldots, N / 2-1 .
$$

A simple calculation leads to

$$
\rho(\delta)=\mathcal{Z}_{L}^{-1} \int \mathcal{D} p w_{\mathrm{b}} \sum_{i}\langle i, i+\delta|\Omega| i, i+\delta\rangle .
$$

Finally, we would like to point out that other observables, such as the total energy and the momentum distribution $\left\langle c_{k \sigma}^{\dagger} c_{k \sigma}\right\rangle$, may also be measured within the current approach, while correlation functions such as $\left\langle\hat{n}_{i} \hat{x}_{j}\right\rangle$ or the quasiparticle weight cannot be determined accurately $\underline{\underline{7}}$ 


\section{Principal components and reweighting}

We make use of the principal component representation and the reweighting procedure, which have been discussed in detail in I. Defining the principal components $\boldsymbol{\xi}_{i}=A^{1 / 2} \boldsymbol{p}_{i}$, in terms of which $S_{\mathrm{b}}$ [Eq. (9)] takes a Gaussian form which can be sampled exactly, ${ }^{7}$ allows to perform calculations that are free of any autocorrelations between successive phonon configurations. In combination with the reweighting, every new phonon configuration is accepted, and measurements can be made after each sweep through the $N \times L$ space-time lattice. The reweighting refers to the use of the purely bosonic weight $w_{\mathrm{b}}$ in the QMC simulation, while all the influence of the electrons and their interaction with the phononscontained in $w_{\mathrm{f}}$-is treated exactly as part of the observables.

\section{Numerical details and performance}

The most significant difference between the present calculations and the one-electron case in I is the dimension of the matrices involved. While for one electron all matrices have size $N \times N-N$ being the extension of the 1D lattice under consideration - here the dimension is $N^{2} \times N^{2}$. Clearly, this restricts calculations with respect to the number of lattice sites, especially in higher dimensions $\mathrm{D}>1$ where $N^{2} \mapsto N^{2 \mathrm{D}}$. The total numerical effort for the current approach is proportional to $N^{6 \mathrm{D}} L$. In contrast, the one-electron algorithm ${ }^{7}$ displays the same dependence $\propto N^{3 \mathrm{D}} L$ as the determinant QMC method of Blankenbecler et al ${ }^{33}$ for the many-electron case, which can be reduced to $N^{2 \mathrm{D}} L$ by employing the checkerboard breakup of the hopping matrix ${ }^{32}$ The increase in required computer time for the bipolaron results from the fixed number of electrons. Recently, a grandcanonical version of the one-electron algorithm, also with a computer time $\sim N^{2 \mathrm{D}} L$, has been applied to study the dependence of polaron formation on carrier density in the spinless Holstein model ${ }^{34}$ For the bipolaron problem, we shall see below that the present algorithm allows one to study lattices of reasonable size $N \leq 14$, for a wide range of the parameters $\bar{\omega}, \lambda$ and $\bar{U}$. In particular, we can obtain accurate results in the adiabatic regime $\bar{\omega}<1$.

Let us briefly compare our method to other QMC approaches to the HH bipolaron. The method of de Raedt and Lagendijk ${ }^{28}$ is based on an analytic integration over the phonon degrees of freedom, leading to a model with retarded el-el interaction. Similar to our approach, it employs a Suzuki-Trotter approximation and gives results at finite temperatures. For simplicity, de Raedt and Lagendijk only considered the adiabatic limit $\bar{\omega}=0$, in which there are no retardation effects. The numerical effort grows as $L^{2}$, but is virtually independent of the system size, so that simulations can be carried out even for large clusters in three dimensions. However, it is not clear how a small but finite phonon frequency $\bar{\omega}<1$ will affect the computer time.

Macridin et al ${ }^{29}$ used the diagrammatic QMC method to study two electrons on a $25 \times 25$ lattice. Although their approach does not rely on the Suzuki-Trotter decomposition, it is limited to zero temperature, and statistical errors increase noticeably for $\bar{\omega}<1$. Moreover, the accuracy also decreases for large values of $\lambda$ and/or $\bar{U}$, whereas we shall see in Sec. VI that we can easily study the strong el-ph coupling regime also for $\bar{U}>0$.

In I, we announced the possibility of reducing the numerical effort for the present method by exploiting the translational invariance of the model. To this end, the basis states (11) would have to be replaced by states $\{|k, \Delta\rangle\}$ with total quasimomentum $k$, and with the two electrons separated by a distance $\Delta$. A similar idea has been used by Kornilovitch ${ }^{35,36}$ for a single electron. In one dimension, the use of the basis $\{|k, \Delta\rangle\}$ would reduce the size of the matrices in the algorithm from $N^{2} \times N^{2}$ to $N \times N$. However, in the course of the simulation, we had to evaluate the matrix product over $\tau$ [Eq. (15)] for each allowed value of $k$. In total, we could therefore reduce the numerical effort by a factor $N$. The major drawback of using the reduced basis in momentum space is that it significantly complicates the program code. Consequently, in this work, we have restricted ourselves to the straight-forward extension of the one-electron algorithm presented in I.

Finally, the minus-sign problem, which has been mentioned in I, also exists here. However, as for one electron, it quickly diminishes with increasing system size, and does therefore not conceivably affect simulations.

\section{VARIATIONAL APPROACH}

Although the method can easily be applied also in higher dimensions, we wish to keep the notation simple and therefore restrict the derivation to $\mathrm{D}=1$. The approximation consists of the use of a zero-phonon basis after the extended unitary transformation, which leads to $\tilde{I}_{\text {ep }}=0$ [Eq. (2)]. Furthermore, neglecting the groundstate energy of the oscillators, we also have $P=0$, so that

$$
\tilde{H}=\tilde{K}+\tilde{I}_{\mathrm{ee}},
$$

with the transformed hopping term

$$
\tilde{K}=-t_{\mathrm{eff}} \sum_{\langle i j\rangle \sigma} c_{i \sigma}^{\dagger} c_{j \sigma}=\sum_{k \sigma} \varepsilon(k) c_{k \sigma}^{\dagger} c_{k \sigma}
$$

and $\varepsilon(k)=-2 t_{\text {eff }} \sum_{k \sigma} \cos (k)$. Here, the effective hopping amplitude is given by $\underline{\underline{\underline{T}}}$

$$
t_{\mathrm{eff}}=\frac{1}{z} \sum_{\delta} e^{-\frac{1}{4} \sum_{l}\left(\gamma_{l-\delta}-\gamma_{l}\right)^{2}},
$$

where $\delta= \pm 1$ in one dimension, $z$ is the number of nearest neighbors, and rotational invariance has been ex- 
ploited. For two electrons of opposite spin, the interaction term (3) simplifies to

$$
\tilde{I}_{\mathrm{ee}}=2 v_{0}-U+2 \sum_{i j} v_{i j} \hat{n}_{i \uparrow} \hat{n}_{j \downarrow}
$$

if we use $v_{i j}=v_{|j-i|}$ and $\hat{n}_{i \sigma} \hat{n}_{j \sigma}=0$ for $i \neq j$. The two-electron eigenstates of the Hamiltonian (23) have the form

$$
\left|\psi_{k}\right\rangle=\sum_{p} \tilde{d}_{p} c_{k-p \downarrow}^{\dagger} c_{p \uparrow}^{\dagger}|0\rangle
$$

Here we have suppressed the phonon component which is simply given by the ground state of $N$ free harmonic oscillators. The states (27) may be written as

$$
\left|\psi_{k}\right\rangle=\frac{1}{\sqrt{N}} \sum_{i} e^{\mathrm{i} k x_{i}} \sum_{l} d_{l} c_{i \downarrow}^{\dagger} c_{i+l \uparrow}^{\dagger}|0\rangle
$$

where the Fourier transform

$$
\boldsymbol{d}=F \tilde{\boldsymbol{d}}
$$

with $F_{l p}=e^{\mathrm{i} x_{l} p} / \sqrt{N}$ has been employed. The normalization of Eq. (27) reads $\left\langle\psi_{k} \mid \psi_{k}\right\rangle=\sum_{p}\left|d_{p}\right|^{2}$.

The expectation value of the transformed hopping term with respect to the states defined by Eq. (27) becomes

$$
\begin{aligned}
\left\langle\psi_{k}|\tilde{K}| \psi_{k}\right\rangle & =\sum_{p p^{\prime}} \tilde{d}_{p}^{*} \tilde{d}_{p^{\prime}} \sum_{q} \varepsilon(q)(\underbrace{\left\langle 0\left|c_{p \uparrow} c_{k-p \downarrow} \hat{n}_{q \uparrow} c_{k-p^{\prime} \downarrow}^{\dagger} c_{p^{\prime} \uparrow}^{\dagger}\right| 0\right\rangle}_{\delta_{p, p^{\prime}} \delta_{q, p}}+\underbrace{\left\langle 0\left|c_{p \uparrow} c_{k-p \downarrow} \hat{n}_{q \downarrow} c_{k-p^{\prime} \downarrow}^{\dagger} c_{p^{\prime} \uparrow}^{\dagger}\right| 0\right\rangle}_{\delta_{p, p^{\prime}} \delta_{q, k-p}}) \\
& =\sum_{p}\left|\tilde{d}_{p}\right|^{2}[\varepsilon(p)+\varepsilon(k-p)]=-4 t_{\mathrm{eff}} \boldsymbol{d}^{\dagger} T_{k} \boldsymbol{d} .
\end{aligned}
$$

In the last step we introduced vector notation, defined $T_{k}=F \operatorname{diag}[\cos (p)+\cos (k-p)] / 2 F^{\dagger}$ and used Eq. (29). The expectation value of the interaction term is best computed in the real-space representation (28). We find

$$
\begin{aligned}
\left\langle\psi_{k}\left|\tilde{I}_{\mathrm{ee}}\right| \psi_{k}\right\rangle & =\left(2 v_{0}-U\right) \sum_{l}\left|d_{l}\right|^{2}+\frac{2}{N} \sum_{i j} v_{i j} \sum_{j^{\prime} j^{\prime \prime}} \sum_{l l^{\prime}} d_{l}^{*} d_{l^{\prime}} e^{\mathrm{i} k\left(x_{l}-x_{l^{\prime}}\right)} \underbrace{\left\langle 0\left|c_{j^{\prime}+l \uparrow} c_{j^{\prime} \downarrow} \hat{n}_{i \uparrow} \hat{n}_{j \downarrow} c_{j^{\prime \prime} \downarrow}^{\dagger} c_{j^{\prime \prime}+l^{\prime} \uparrow}^{\dagger}\right| 0\right\rangle}_{\delta_{j j^{\prime}} \delta_{j j^{\prime \prime}} \delta_{i, j+l} \delta_{l, l^{\prime}}} \\
& =\left(2 v_{0}-U\right) \sum_{l}\left|d_{l}\right|^{2}+\frac{2}{N} \sum_{j, l} v_{j+l, j}\left|d_{l}\right|^{2}=\left(2 v_{0}-U\right) \boldsymbol{d}^{\dagger} \boldsymbol{d}+2 \boldsymbol{d}^{\dagger} V \boldsymbol{d},
\end{aligned}
$$

where the diagonal matrix $V_{i j}=\delta_{i j} v_{i}$ has been introduced. The minimization with respect to $\boldsymbol{d}$ yields the eigenvalue problem

$$
\left(-4 t_{\mathrm{eff}} T_{k}+2 V\right) \boldsymbol{d}=\left(E_{0}-2 v_{0}+U\right) \boldsymbol{d}
$$

The vector of coefficients $\boldsymbol{d}$ and thereby the ground state are determined by minimizing the ground-state energy $E_{0}$ through variation of the displacement fields $\gamma_{i j}$. In the present work, we use the unconstrained nonlinear optimization routine fminsearch from the MATLAB package, together with several different starting points, including the simple LF result and random values of the $\gamma_{i j}$. This ensures reproducible results even for a large number of variational parameters.

In contrast to the local LF transformation, this procedure takes into account displacements of the oscillators not only at the same but also at the sites surrounding the two electrons. This represents a physically much better ansatz to describe the extended state which exists for weak el-ph coupling and/or strong Coulomb repulsion. Similar to the one-electron problem, we shall refer to the result obtained from the above variational method by replacing $\gamma_{i j}$ with $\gamma \delta_{i j}$ as the Holstein Lang-Firsov (HLF) approximation.

\section{RESULTS}

Before we turn to the results, we would like to review briefly the physics of the one-dimensional HH bipolaron as it emerges from existing work (see Sec. III). In the absence of Coulomb repulsion, the two electrons form a bound state for any $\lambda>0$. A crossover from an extended state, also called a large bipolaron, to a small bipolaronwith both electrons occupying the same site-is observed at a critical coupling strength $\lambda_{\mathrm{c}}$. The value of $\lambda_{\mathrm{c}}$ is determined by the competition between the different terms in the Hamiltonian (1). Similar to the one-electron case, for small phonon frequencies, the crossover takes place when the gain in potential energy due to bipolaron formation overcomes the loss in kinetic energy. While the former can be estimated in the atomic limit as $4 E_{\mathrm{P}}$ (see, e.g., Ref. 9), the latter is given here by $W=4 t$ - the ki- 
TABLE I: Conditions for the existence of different singlet bipolaron states (see text). Here "wc" and "sc" denote weak coupling and strong coupling, respectively.

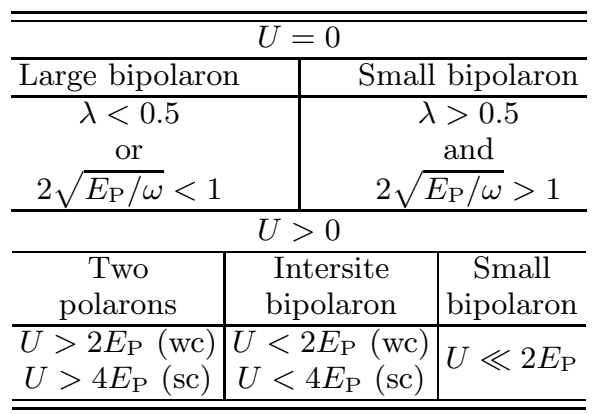

netic energy of the two electrons at $\lambda=0$. Since $\lambda$ can also be written in the form $\lambda=\frac{1}{2}\left(4 E_{\mathrm{P}} / W\right)$, we expect $\lambda_{\mathrm{c}}=0.5$. For larger phonon frequencies $\bar{\omega} \gg 1$, the lattice energy plays an important role, and gives rise to the additional criterion $2 \sqrt{E_{\mathrm{P}} / \omega}>1$ for the existence of a small bipolaron ${ }^{\underline{5}}$

For $\bar{U}>0$, a state with two weakly bound polarons is stable for weak enough el-ph interaction. Interestingly, starting from a small bipolaron a cross over to an intersite bipolaron - with the two electrons being localized most likely at neighboring lattice sites - takes place at a critical value $\bar{U}_{\mathrm{c}} \cdot 13.24 .25$ This state has been shown to have a much smaller effective mass than an on-site bipolaron, 25 and may therefore exist as a mobile quasiparticle in real systems. Phase diagrams of the intersite bipolaron have been reported in one ${ }^{24.25}$ and two dimensions ${ }^{29}$ For $\bar{\omega}=$ 1 , the region where such a state exists in one dimension is quite accurately described by $U<2 E_{\mathrm{P}}$ at weak el-ph coupling, and by $U<4 E_{\mathrm{P}}$ at strong coupling, ${ }^{25}$

If $2 E_{\mathrm{P}} \gg U$ the two electrons can overcome the on-site repulsion and form a small bipolaron. As we shall see below, the competition between Hubbard repulsion and attractive interaction due to the electron-lattice coupling depends critically on the phonon frequency. A summary of the different bipolaron states together with (approximate) conditions for their existence is given in Tab. [

We would like to mention that very interesting physics can also be deduced from the bipolaron band dispersion (see Ref. 9 and references therein). Although the latter can be studied in principle within our variational approach, the subtle effects originating from the retarded nature of the el-ph interaction ${ }^{9}$ could not be addressed in a satisfactory way.

\section{A. Quantum Monte Carlo}

To eliminate the error $\sim(\Delta \tau)^{2}$ due to the SuzukiTrotter approximation, we extrapolate the QMC results to $\Delta \tau=0$. In contrast, this error is expected to be
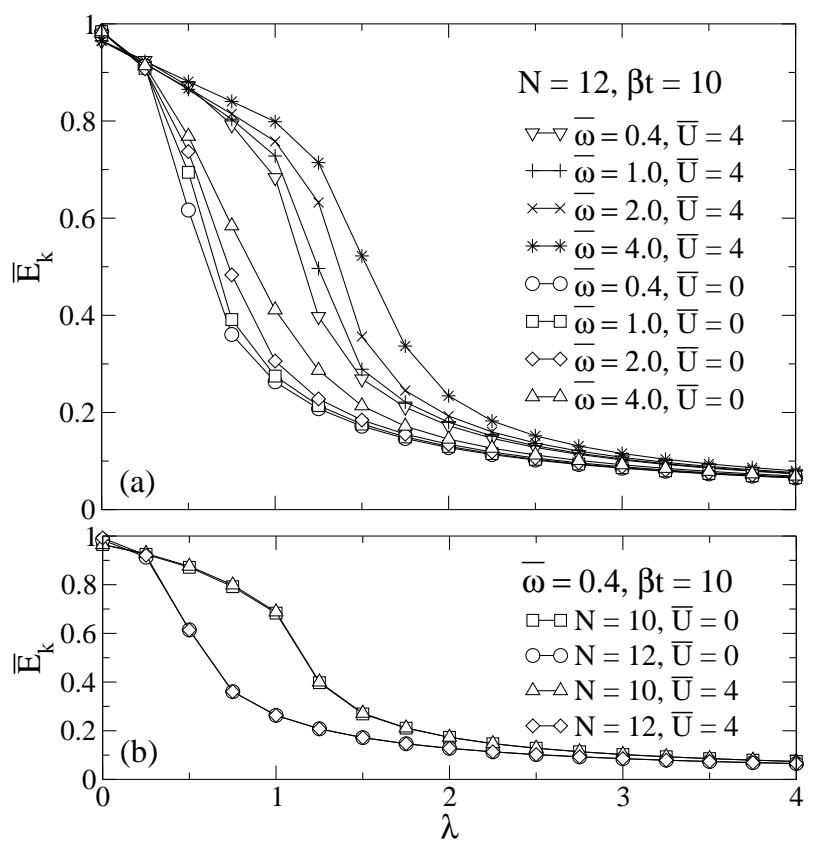

FIG. 1: (a) Normalized kinetic energy $\bar{E}_{\mathrm{k}}$ [Eq. (31)] from QMC as a function of the el-ph coupling $\lambda$ for different values of the phonon frequency $\bar{\omega}$ and the Hubbard repulsion $\bar{U}$. (b) Dependence of $\bar{E}_{\mathrm{k}}$ on the cluster size $N$. Here and in subsequent figures QMC results have been extrapolated to $\Delta \tau=0$ (see text), errorbars are suppressed if smaller than the symbol size, and lines are guides to the eye.

relatively large (on the order of a few percent) in the calculations of de Raedt and Lagendijk, due to the use of a rather small number of Trotter slices $(L=32$ at $\beta=5$, so that $\Delta \tau \approx 0.16$; see Ref. 28). Here we have performed simulations for three different values of $\Delta \tau$, typically 0.1 , 0.075 and 0.05 . The errorbars in the figures below are usually as small as the linewidth, and will not be shown if smaller than the symbols used.

Owing to the increased numerical effort compared to the one-electron case, $\underset{7}{\underline{7}}$ we shall only present results for $N \leq 12$. Fortunately, finite-size effects on, e.g., the kinetic energy, are already very small for this cluster size, as illustrated by Fig. 1(b) for the most critical parameters. As expected, the largest changes with $N$ occur near the crossover to a small bipolaron. ${ }^{7}$ Similar behavior has been found for the correlation function $\rho(\delta)$.

We define the effective kinetic energy of the two electrons as

$$
\bar{E}_{\mathrm{k}}=E_{\mathrm{k}} /(-4 t) .
$$

In Fig. 11a) we depict $\bar{E}_{\mathrm{k}}$ as a function of the el-ph coupling for different values of $\bar{\omega}$ and $\bar{U}$, at $\beta t=10$. This value of the inverse temperature is twice as large as in previous work $\stackrel{28}{2}$ yielding results sufficiently close to the ground state to reveal the effects of bipolaron formation.

Figure丩(a) reveals a strong decrease of $\bar{E}_{\mathrm{k}}$ near $\lambda=0.5$ for small phonon frequencies and $\bar{U}=0$. With increasing $\bar{\omega}$, the crossover becomes less pronounced, and shifts to 
larger values of $\lambda$. For the same value of $\bar{\omega}$, the crossover to a small bipolaron is sharper than the small-polaron crossover in the Holstein model with one electron (see, e.g., I). The small but finite kinetic energy even for strong el-ph interaction is a result of undirected, internal motion of the two electrons inside the phonon cloud. For a finite on-site repulsion $\bar{U}=4, \bar{E}_{\mathrm{k}}$ remains fairly large up to $\lambda \approx 1$ (for $\bar{\omega} \lesssim 2.0$ ), in agreement with the strongcoupling result $\lambda_{\mathrm{c}}=1$ for $\bar{U}=4$ (see discussion in Ref. 9 ). At even stronger coupling, the Hubbard repulsion is overcome, and a small bipolaron is formed. Again, we see that the critical coupling increases with increasing phonon frequency. We would like to mention that the kinetic energy has also been calculated by ED on clusters of up to twelve sites, 20.23 .24 but results for $\bar{\omega}<1$ were restricted in the accessible range of $\lambda$. In the regime where ED is applicable, a very good agreement has been found with our QMC data.

The nature of the bipolaron state is revealed by the correlation function $\rho(\delta)$ defined in Eq. 21), which gives the probability for the two electrons to be separated by a distance $\delta \geq 0$, and therefore represents a direct measure for the size of the bipolaron. Clearly, we have the sum rule $\sum_{\delta} \rho(\delta)=1$. As pointed out, e.g., by Marsiglio, $\frac{19}{\underline{p}}$ the phonon frequency determines the degree of retardation of the el-ph interaction, and thereby sets the maximal allowed distance between the two electrons compatible with a bound state. In the sequel, we shall focus on the most interesting case of small phonon frequencies, which has often been avoided in previous work for reasons outlined in Sec. [1]

Figure 2(a) shows $\rho(\delta)$ as a function of $\lambda$ for $\bar{U}=0$. Starting from the noninteracting state $(\lambda=0)$ with $\rho=$ $1 / N$, we see a pronounced increase of $\rho(0)$ near $\lambda=0.5$. For large $\lambda \gtrsim 2$, we have $\rho(0) \approx 1$ and $\rho(\delta) \approx 0$ for $\delta>0$, characteristic for the on-site bipolaron. The decrease of the spatial extent of the bipolaron with increasing el-ph interaction is better illustrated in the inset of Fig. 22(a), where we depict $\rho$ as a function of $\delta$. For finite on-site repulsion $\bar{U}=4$, an extended bipolaron state is stabilized for small $\lambda$ [Fig. 2(b)], while a small bipolaron is found for $\lambda=2$. Additionally, we see that for $\lambda=1$, the electrons are most likely to occupy neighboring lattice sites [intersite bipolaron, see also inset in Fig. [2(b)].

As pointed out earlier, a crossover from a small to an intersite bipolaron to two weakly bound polarons takes place as a function of the Hubbard interaction. Since the latter competes with the retarded el-ph interaction, the phonon frequency is expected to be an important parameter. In Fig. 3 we show the kinetic energy and the correlation function $\rho(\delta)$ as a function of $\bar{U}$. We have fixed the el-ph coupling to $\lambda=1$. Starting from a small bipolaron for $\bar{U}=0$ [see Fig. 2(a)], the kinetic energy increases with increasing Hubbard repulsion, equivalent to a reduction of the effective bipolaron mass ${ }^{25.26}$ Although the crossover is slightly washed out by the finite temperature in our simulations, there is a well-conceivable increase in $\bar{E}_{\mathrm{k}}$ up to $\bar{U} \approx 4$, above which the kinetic energy
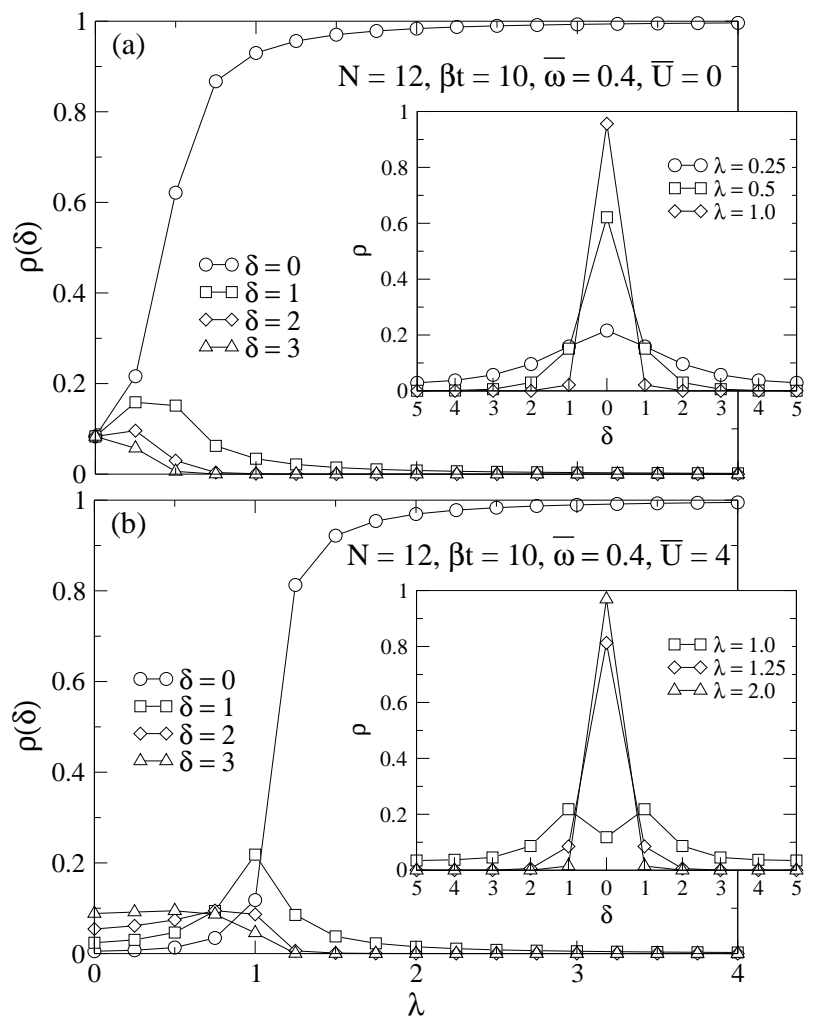

FIG. 2: Correlation function $\rho(\delta)$ [Eq. (21)] from QMC as a function of el-ph coupling $\lambda$ for different values of $\delta$. [(a) $\bar{U}=0$, (b) $\bar{U}=4]$. Inset: Correlation function $\rho(\delta)$ as a function of the distance $\delta$ of the electrons for different $\lambda$.

begins to decrease again. The increase of $\bar{E}_{\mathrm{k}}$ originates from the breakup of the small bipolaron, as indicated by the decrease of $\rho(0)$ in Fig. 3(b). Close to $\bar{U}=4$, the curves for $\rho(0)$ and $\rho(1)$ cross, and it becomes more favorable for the two electrons to reside on neighboring sites. The intersite bipolaron only exists below a critical Hubbard repulsion $U_{\mathrm{c}}$. As discussed at the beginning of this section, the latter is given by $U_{\mathrm{c}}=2 E_{\mathrm{P}}$ (i.e., here $\left.\bar{U}_{\mathrm{c}}=4\right)$ at weak el-ph coupling, and by $U_{\mathrm{c}}=4 E_{\mathrm{P}}$ at strong coupling. For an intermediate value $\lambda=1$ as in Fig. 3. the crossover from the intersite state to two weakly bound polarons is expected to occur somewhere in between, but is difficult to identify exactly from the QMC results.

Figure 3 further illustrates that the crossover becomes steeper with decreasing phonon frequency. In the adiabatic limit $\bar{\omega}=0$, it has been shown to be a first-order phase transition, 15 whereas for $\bar{\omega}>0$ retardation effects suppress any nonanalytic behavior. At the same $\bar{U}, \bar{E}_{\mathrm{k}}$ increases with $\bar{\omega}$ since for a fixed $\lambda$, the bipolaron becomes more weakly bound. For the same reason, the crossover to an intersite bipolaron - showing up in Fig. 3 as a crossing of $\rho(0)$ and $\rho(1)$ - shifts to smaller values of $\bar{U}$.

Let us now consider the effect of temperature. While the kinetic energy shows a similar dependence as in the 


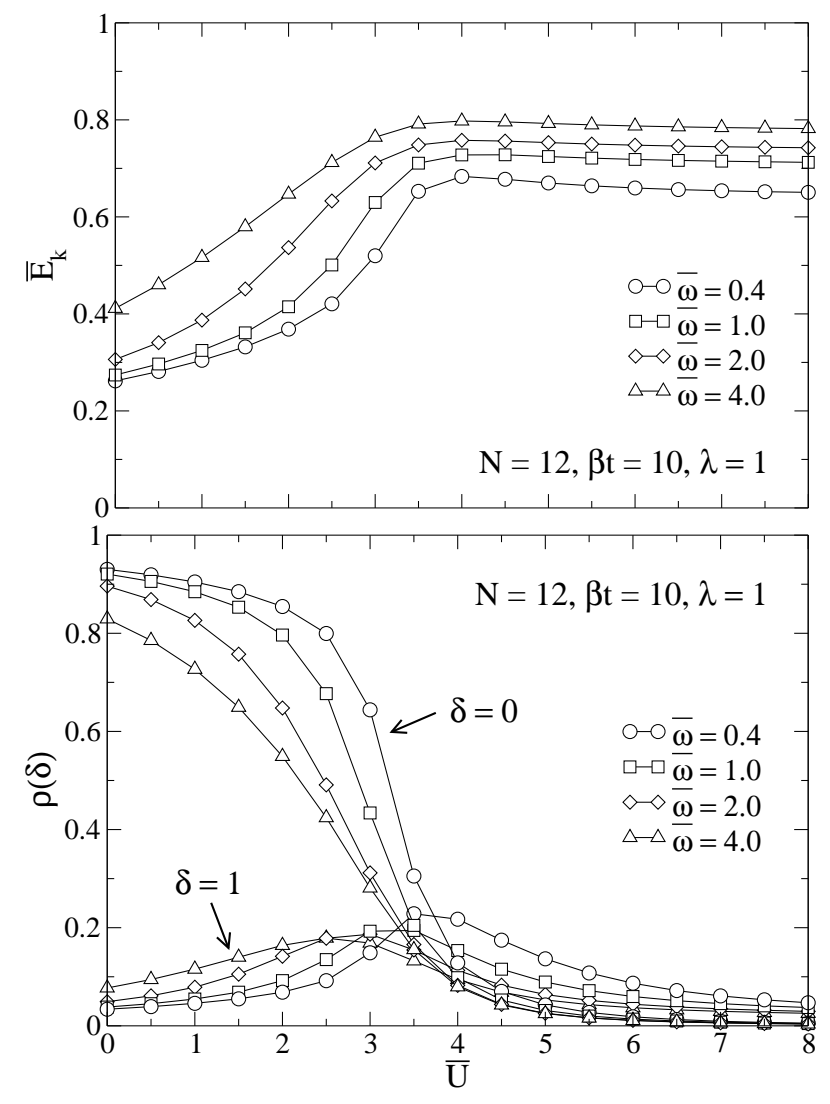

FIG. 3: (a) Normalized kinetic energy $\bar{E}_{\mathrm{k}}$ and (b) correlation functions $\rho(0), \rho(1)$ from QMC as a function of the Hubbard repulsion $\bar{U}$ for different values of the phonon frequency $\bar{\omega}$.

one-electron case - with the crossover being smeared out at high temperatures - it is much more interesting to look at $\rho(\delta)$. In Figs. 4 (a) - (c) we plot $\rho(\delta)$ at different temperatures, for parameters corresponding to the three regimes of a large, small and intersite bipolaron, respectively.

Large bipolaron. For the parameters chosen $(\bar{U}=0$, $\lambda=0.25)$, the two electrons are most likely to occupy the same site, but the bipolaron extends over a distance of several lattice constants [Fig. 4(a)]. Clearly, in this regime, the cluster size $N=12$ used here is not completely satisfactory, but still provides a fairly accurate description as can be deduced from calculations for $N=14$ (not shown). Nevertheless, on such a small cluster, no clear distinction between an extended bipolaron and two weakly bound polarons can be made. As the temperature increases from $\beta t=10$ to $\beta t=1$, the probability distribution broadens noticeably, so that it becomes more likely for the two electrons to be further apart. In particular, for the highest temperature shown, $\rho(0)$ has reduced by about $30 \%$ compared to $\beta t=10$.

Small bipolaron. A different behavior is found for the small bipolaron, which exist at stronger el-ph coupling $\lambda=1.0$. Figure 4 (b) reveals that $\rho(\delta)$ peaks strongly at $\delta=0$, while it is very small for $\delta>0$ at low tempera-
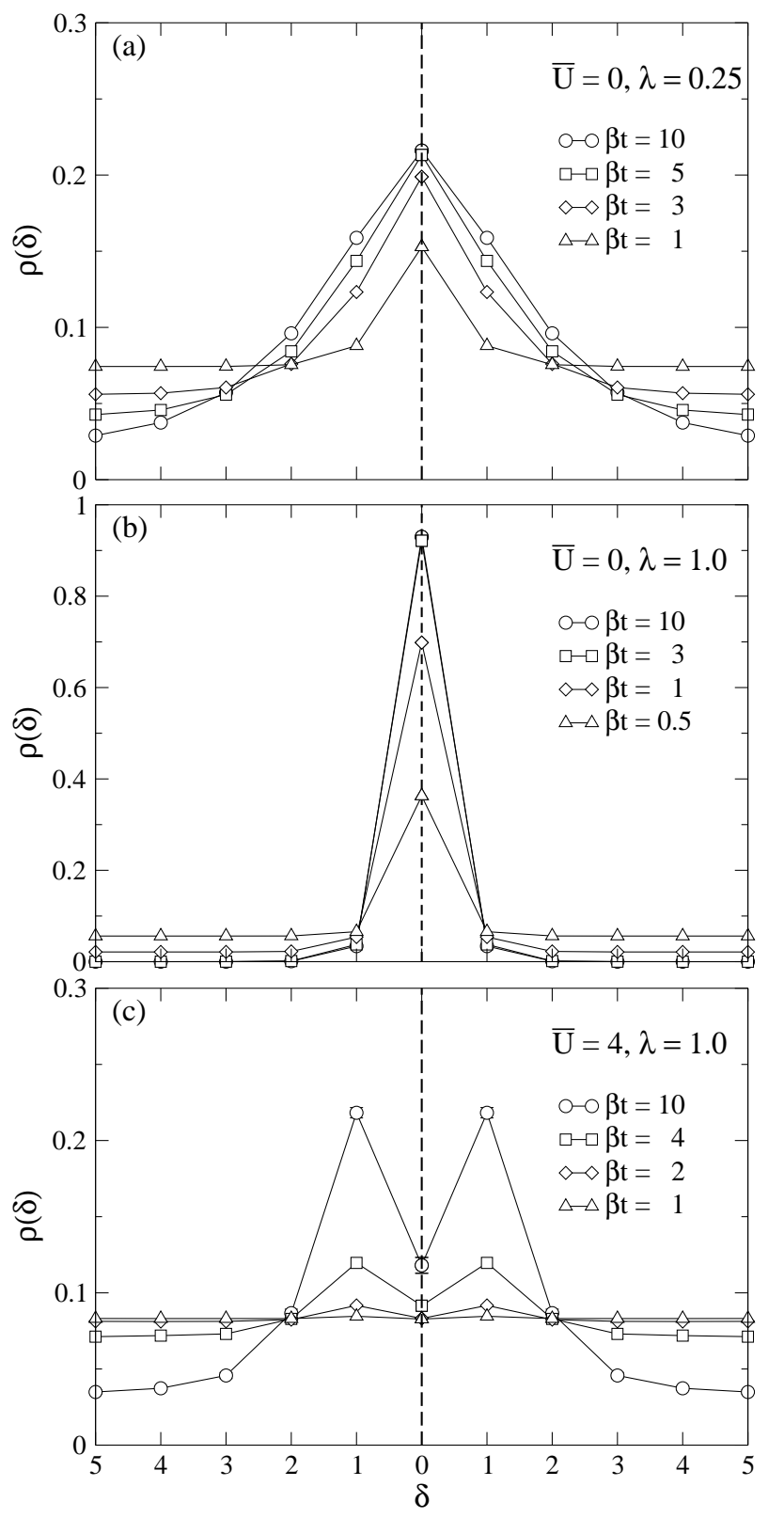

FIG. 4: Correlation function $\rho(\delta)$ from QMC as a function of $\delta$ for different inverse temperatures $\beta, N=12$ and $\bar{\omega}=0.4$.

tures. Increasing the temperature, we observe that $\rho(\delta)$ remains virtually unchanged up to $\beta t=3$. Only at very high temperatures there occurs a noticeable transfer of probability from $\delta=0$ to $\delta>0$. At the highest temperature shown, $\beta t=0.5$, the two electrons have a nonnegligible probability for traveling a finite distance $\delta>0$ apart, although most of the probability is still contained in the peak located at $\delta=0$.

Intersite bipolaron. Finally, we consider in Fig. 4( (c) the intersite bipolaron, which has been found above for $\bar{U}=4$ and $\lambda=1.0$ [Fig. 2(b)]. At low temperatures, $\rho(\delta)$ takes on a maximum for $\delta=1$. For smaller values of $\beta t$, the latter diminishes, until at $\beta t=1$, the distribution is 
completely flat, so that all $\delta$ are equally likely.

The different sensitivity of the bipolaron states to changes in temperature found above can be explained by their different binding energies. The latter is given by $\Delta E_{0}=E_{0}^{(2)}-2 E_{0}^{(1)}$, where $E_{0}^{(1)}$ and $E_{0}^{(2)}$ denote the ground-state energy of the model with one and two electrons, respectively. These quantities can be calculated using the present method as well that presented in I.

Generally, the thermal dissociation is expected to occur at a temperature such that the thermal energy $k_{\mathrm{B}} T=$ $(\beta T)^{-1}$ becomes comparable to $\Delta E_{0}$, in accordance with our numerical data. The large and the intersite bipolaron are relatively weakly bound as a result of the rather small effective interaction $U_{\text {eff }} \approx U-2 E_{\mathrm{P}}$ (see discussion in Ref. 9). The binding energies are $\Delta E_{0} \approx-(0.32 \pm 0.08) t$ and $-(0.28 \pm 0.08) t$, respectively, so that we expect a critical inverse temperature $\beta t \approx 2.5-5, \frac{37}{3}$ in agreement with Figs. 4(a) and (c). In contrast, the small bipolaron in Fig. 4 (b) has a significantly larger binding energy $\Delta E \approx-(3.43 \pm 0.09) t$, and therefore remains stable up to $\beta t \approx 0.3$.

Since the thermal dissociation of intersite bipolarons has been proposed to explain the activated dc conductivity in the paramagnetic state of the manganites, ${ }^{2.3}$ we would like to comment on the relation of our findings to this theory. Instead of the Holstein-type model used here, Alexandrov and Bratkovsky ${ }^{2,3}$ argue in favor of a model with long-range el-ph interaction, and assume that charge carriers are $\mathrm{O}$ p holes rather than $\mathrm{Mn}$ d holes, so that the double-exchange mechanism does not come into play ${ }^{38}$ An intersite bipolaron in their theory is formed by two holes residing on neighboring oxygen ions. Furthermore, they also include a nearest-neighbor Coulomb repulsion $V \approx E_{\mathrm{P}}$ between electrons. In the present case, the latter would, most importantly, reduce the binding energy of the intersite state, thereby leading to a lower temperature for dissociation. For sufficiently large $V$, intersite bipolaron formation is expected to be completely suppressed. A closer investigation of this issue in the framework of the Holstein-Hubbard model may be carried out using a generalization of the present method.

In total, a quantitative comparison of our numerical results to the work of Refs. 2 , and to the 3D manganites, appears to be not justified due to the simplifications made and the different model studied in the present work.

\section{B. Variational approach}

While the above QMC approach is limited to finite temperatures and relatively small clusters, the variational method of Sec. $\nabla$ yields ground-state results on much larger systems. It becomes exact in several limits. First, for $\lambda=0$ (i.e., no el-ph coupling), we obtain the exact solution $\gamma_{i j}=0$ for all $i, j$. Second, as $\omega \rightarrow \infty$, no phonons can be excited so that the use of a zerophonon basis is justified. Similarly, in the classical limit $\omega=0$, the phonons do not have any dynamics, and the

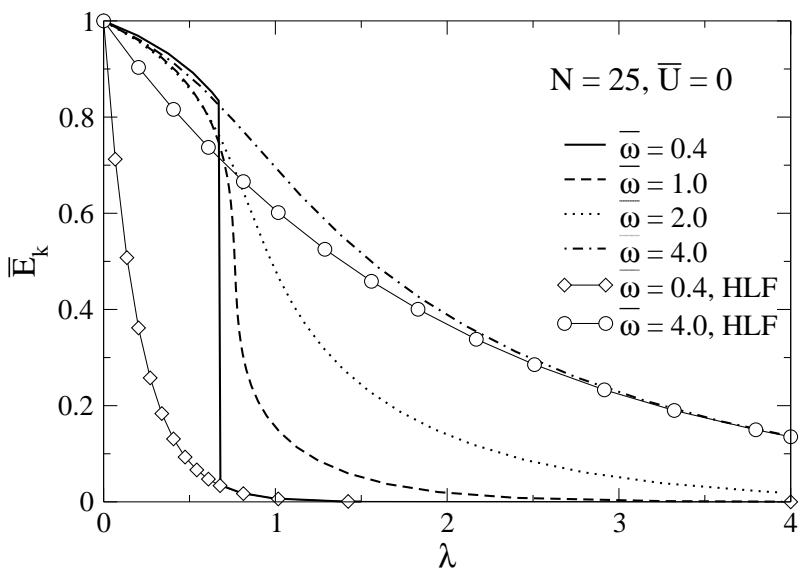

FIG. 5: Variational results for the normalized kinetic energy $\bar{E}_{\mathrm{k}}$ as a function of the el-ph coupling $\lambda$, and for different phonon frequencies $\bar{\omega}$. Also shown are results of the HLF approximation (see text).

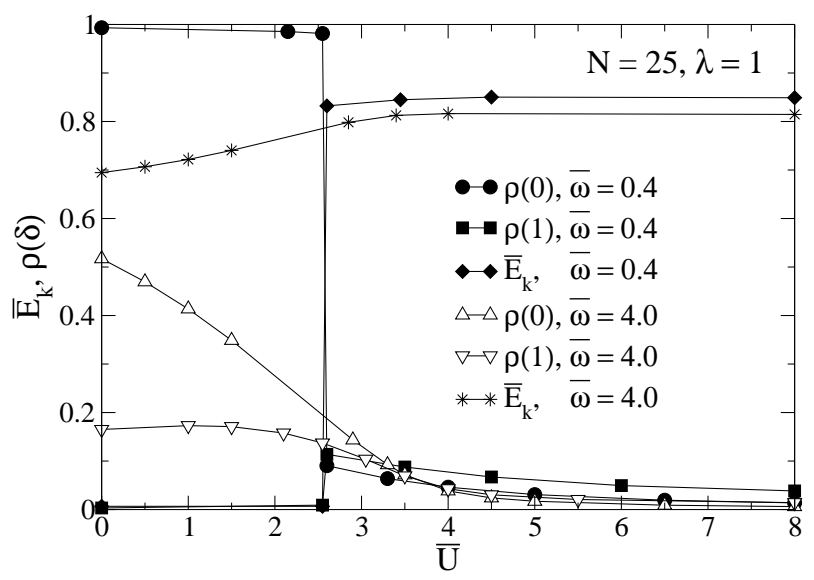

FIG. 6: Variational results for the normalized kinetic energy $\bar{E}_{\mathrm{k}}$ and the correlation functions $\rho(0), \rho(1)$ as a function of the on-site repulsion $\bar{U}$.

variational determination of the displacement fields allows one to obtain exact results for any $\lambda$. In contrast, the HLF approximation (see Sec. V) generally overestimates the displacement of the lattice at a given site in the presence of an electron, even for $\omega=\infty$. Finally, the variational approach becomes exact in the nonadiabatic strong-coupling limit $\lambda, \omega \rightarrow \infty$. Since the two-electron problem is diagonalized exactly without phonons, the above statements hold for any value of the Hubbard repulsion $U$.

To scrutinize the quality of the variational method, we started by comparing the ground-state energy for $\bar{U}=0$ as a function of el-ph coupling for different values of $\bar{\omega}$, to the most accurate approach currently available in one dimension, namely the variational diagonalization. ${ }^{25} \mathrm{We}$ find a good agreement over the whole range of $\lambda$. As expected from the nature of the approximation, slight deviations occur for $\bar{\omega} \lesssim 1$. 
Despite the success in calculating the total energybeing the quantity that is optimized - one has to be careful not to overestimate the validity of any variational method. To reveal the shortcomings of the current approach, we show in Fig. 5] the normalized kinetic energy $\bar{E}_{\mathrm{k}}=t_{\mathrm{eff}}$ [see Eqs. (25) and (31)] as a function of el-ph coupling, and for different $\bar{\omega}$. We have chosen $N=25$ to ensure negligible finite-size effects. In principle, Fig. 5 displays a behavior similar to the QMC data in Fig. 11(a). There is a strong reduction of $\bar{E}_{\mathrm{k}}$ near $\lambda=0.5$ for $\bar{\omega}=0.4$, which becomes washed out and moves to larger $\lambda$ with increasing phonon frequency. Compared to the exact QMC results in Fig. 1(a), the crossover to a small bipolaron is much too steep in the adiabatic regime, regardless of the fact that the variational results are for $T=0$. This is a common defect of variational methods. Moreover, for $\bar{\omega}=0.4-2.0$, the variational kinetic energy is too small above the bipolaron crossover compared to the QMC data, while for $\bar{\omega}=4$, the decay of $\bar{E}_{\mathrm{k}}$ with increasing $\lambda$ is too slow.

The reason for the failure is the absence of retardation effects, which play a dominant role in the formation of bipolaron states. The increased importance of the phonon dynamics - not included in the variational method-for the two-electron problem leads to a less good agreement with exact results than in the oneelectron case $\stackrel{7}{I n}$ particular, our variational results overestimate the position of the crossover (Fig. 5) compared to the value $\lambda_{c}=0.5$ expected in the adiabatic regime. Nevertheless, the method represents a significant improvement over the simple HLF approximation, due to the variational determination of the parameters $\gamma_{i j}$. This is illustrated in Fig. 5 where we also show the HLF result $\bar{E}_{\mathrm{k}}=\exp \left(-E_{\mathrm{P}} / \omega\right)$ for $\bar{\omega}=0.4$ and 4.0 . In contrast to the variational approach, the HLF approximation yields an exponentially decaying kinetic energy for all values of the phonon frequency. Whereas such behavior actually occurs in the nonadiabatic limit $\omega \rightarrow \infty$, the situation is different for small $\bar{\omega}$ [see Figs. 11(a) and 5]. Thus the HLF approach cannot reproduce the physics of bipolaron formation for small and intermediate phonon frequencies, while the variational method presented here accounts qualitatively for the dependence on the phonon frequency.

Next, we wish to study the influence of Coulomb repulsion $\bar{U}$. Similar to Fig. [3] we take $\lambda=1$, so that an on-site bipolaron state is formed at $\bar{U}=0$. For small phonon frequency $\bar{\omega}=0.4$, Fig. 6] reveals a sharp crossover near $\bar{U}=2.5$, i.e., at a smaller value of $\bar{U}$ than in the QMC results of Fig. 3 the reason again being the neglect of the retarded nature of the effective el-el interaction. As in the QMC results, the Coulomb repulsion breaks up the on-site bipolaron, leading to an increase of the kinetic energy. Moreover, the curve for $\rho(1)$ peaks at the crossover point, indicating the existence of an intersite bipolaron in this regime. A similar picture is found for larger phonon frequency $\bar{\omega}=4$, also shown in Fig. 6] although the changes with increasing $\bar{U}$ are much more
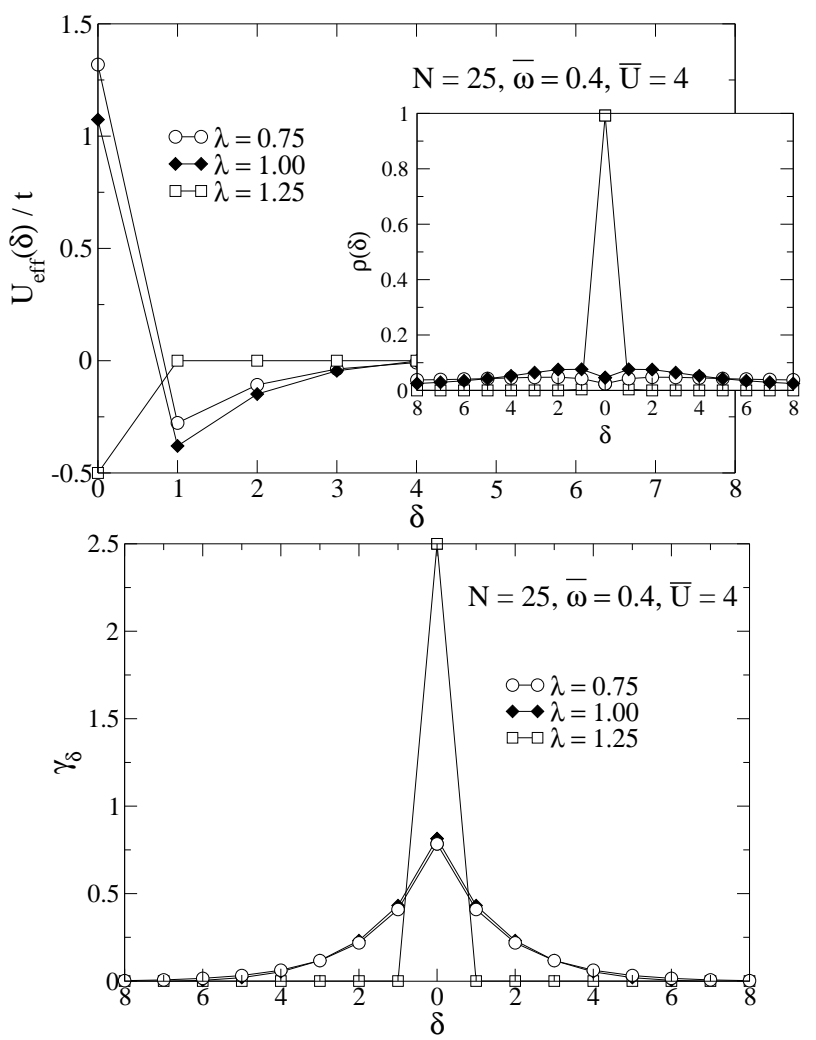

FIG. 7: Upper panel: variational results for the effective interaction $U_{\text {eff }}(\delta)$ (see text) and the correlation function $\rho(\delta)$ (inset) as a function of the el-el distance $\delta$. Lower panel: variational lattice distortions $\gamma_{\delta}$ as a function of $\delta$.

gradual than for $\bar{\omega}=0.4$.

Finally, we report in Fig. 7] (upper panel) the effective interaction $U_{\text {eff }}(\delta)$ between the two electrons as a function of their relative distance $\delta$, given by $v_{\delta}$ [Eq. (3)]. We have chosen $\bar{\omega}=0.4$ and $\bar{U}=4$, the same parameters as in Fig. 2(b). For $\lambda=0.75$, the finite Coulomb repulsion stabilizes two weakly bound polarons, as illustrated by the results for $\rho(\delta)$ shown in the inset of Fig. [7] While $U_{\text {eff }}$ is repulsive (positive) for $\delta=0$, the two electrons can form a bound state by traveling a finite distance $1 \leq \delta \lesssim 4$ apart. This is still true for $\lambda=1$, for which the HLF approach yields $U_{\text {eff }}(0)=U-2 E_{\mathrm{P}}=0$. Nevertheless, the two electrons experience an attractive interaction and form an extended bipolaron. Finally, for even stronger coupling $\lambda=1.25$, the phonon-mediated elel interaction has overcome the on-site repulsion, so that $U_{\text {eff }}(\delta=0)<0$. At the same time, the size of the bipolaron has collapsed to a single site. This crossover is also well visible in the lower panel, which displays the variationally determined lattice distortions $\gamma_{\delta}$. It is worth mentioning that the values of $U_{\text {eff }}(0)$ in Fig. 7 are larger than the strong-coupling prediction $U-2 E_{\mathrm{P}}$ for all values of $\lambda$ considered. This may be attributed to the overestimated bipolaron binding energy in the atomic limit.

As pointed out in several places, the shortcomings of the variational approach presented here are a result of the 
missing dynamical phonon effects. The present approach may be further improved by making an ansatz for the eigenstates of the untransformed Hamiltonian (11) of the form

$$
\begin{aligned}
\left|\Psi_{k}\right\rangle=\frac{1}{N} \sum_{i j} & \sum_{p} e^{\mathrm{i} p x_{i}+(k-p) x_{j}} \\
& \times\left(\tilde{d}_{p}^{(1)} \nu^{\dagger}\left\{\gamma^{(1)}\right\}+\tilde{d}_{p}^{(2)} \nu^{\dagger}\left\{\gamma^{(2)}\right\}\right)|i, j\rangle
\end{aligned}
$$

with $|i, j\rangle$ defined as in Eq. (11), two canonical transformations depending on the displacement fields $\gamma_{i j}^{(1)}$ and $\gamma_{i j}^{(2)}$ (see Sec. III), and additional variational parameters $\tilde{d}_{p}^{(1)}, \tilde{d}_{p}^{(2)}$. Thereby, one can take into account lattice distortions not centered at the sites of the electrons, which become important as $\bar{\omega} \rightarrow 0$, and which reproduce to some degree the effect of retardation.

\section{CONCLUSIONS}

We have studied the Holstein-Hubbard bipolaron with quantum phonons by extending a quantum Monte Carlo method previously developed for the Holstein polaron ${ }^{7}$ In its present form, the method is limited to onedimensional clusters. However, in contrast to other approaches, it allows to perform accurate calculations also for small phonon frequencies and finite temperatures.

We have studied the dependence of bipolaron formation on the phonon frequency and the Hubbard repulsion.
Our results underline the importance of the phonon dynamics, which has often been neglected in previous work. Moreover, we have presented for exact results for the effect of temperature on the bipolaron state in the important adiabatic regime. Thermal dissociation of bipolarons has been observed at temperatures where the thermal energy becomes comparable to the binding energy.

Two interesting open issues are the effect of nearestneighbor Coulomb interaction, as well as that of dimensionality. While the latter cannot easily be addressed with the current approach, one may instead extend the promising work of Ref. 28 to finite phonon frequencies.

Finally, we have proposed a variational ansatz based on a canonical transformation with variational parameters. The latter represents a significant improvement over standard approximations. In particular, it qualitatively accounts for the dependence on the phonon frequency.

\section{Acknowledgments}

This work has been supported by the Austrian Science Fund (FWF), project No. P15834. One of us (M. H.) is indebted to DOC (Doctoral Scholarship Program of the Austrian Academy of Sciences). We would like to thank Holger Fehske for useful discussion and valuable comments on the manuscript.
* Electronic address: hohenadler@itp.tu-graz.ac.at

1 A. S. Alexandrov, V. V. Kabanov, and N. F. Mott, Phys. Rev. Lett. 77, 4796 (1996).

2 A. S. Alexandrov and A. M. Bratkovsky, Phys. Rev. Lett. 82, 141 (1999).

3 A. S. Alexandrov and A. M. Bratkovsky, J. Phys.: Condens. Matter 11, 1989 (1999).

4 B. K. Chakraverty, J. Ranninger, and D. Feinberg, Phys. Rev. Lett. 81, 433 (1998).

5 B. K. Chakraverty, J. Ranninger, and D. Feinberg, Phys. Rev. Lett. 82, 2621 (1999).

6 D. M. Edwards, Adv. Phys. 51, 1259 (2002).

7 M. Hohenadler, H. G. Evertz, and W. von der Linden, Phys. Rev. B 69, 024301 (2004).

8 T. Holstein, Ann. Phys. (N.Y.) 8, 325; 8, 343 (1959).

9 M. Hohenadler, M. Aichhorn, and W. von der Linden, Phys. Rev. B 71, 014302 (2005).

10 A. S. Alexandrov and N. Mott, Polaron 64 Bipolarons (World Scientific, Singapore, 1995).

11 M. H. Cohen, E. N. Economou, and C. M. Soukoulis, Phys. Rev. B 29, 4496 (1984).

12 D. Emin, J. Ye, and C. L. Beckel, Phys. Rev. B 46, 10710 (1992).

13 A. La Magna and R. Pucci, Phys. Rev. B 55, 14886 (1997).

14 L. Proville and S. Aubry, Physica D 113, 307 (1998).

15 L. Proville and S. Aubry, Eur. Phys. J. B 11, 41 (1999).
16 L. Proville and S. Aubry, Eur. Phys. J. B 15, 405 (2000).

17 G. De Filippis, V. Cataudella, G. Iadonisi, V. Marigliano Ramaglia, C. A. Perroni, and F. Ventriglia, Phys. Rev. B 64, 155105 (2001).

18 J. Ranninger and U. Thibblin, Phys. Rev. B 45, 7730 (1992).

19 F. Marsiglio, Physica C 244, 21 (1995).

20 G. Wellein, H. Röder, and H. Fehske, Phys. Rev. B 53, 9666 (1996).

21 E. V. L. de Mello and J. Ranninger, Phys. Rev. B 55, 14 872 (1997).

22 E. V. L. de Mello and J. Ranninger, Phys. Rev. B 58, 9098 (1998).

23 H. Fehske, H. Röder, G. Wellein, and A. Mistriotis, Phys. Rev. B 51, 16582 (1995).

24 A. Weiße, H. Fehske, G. Wellein, and A. R. Bishop, Phys. Rev. B 62, R747 (2000).

25 J. Bonča, T. Katrašnik, and S. A. Trugman, Phys. Rev. Lett. 84, 3153 (2000).

26 S. El Shawish, J. Bonča, L. C. Ku, and S. A. Trugman, Phys. Rev. B 67, 014301 (2003).

27 C. Zhang, E. Jeckelmann, and S. R. White, Phys. Rev. B 60, 14092 (1999).

28 H. De Raedt and A. Lagendijk, Z. Phys. B: Condens. Matter 65, 43 (1986).

29 A. Macridin, G. A. Sawatzky, and M. Jarrell, Phys. Rev. 
B 69, $245111(2004)$

30 J. Bonča and S. A. Trugman, Phys. Rev. B 64, 094507 (2001).

31 I. G. Lang and Y. A. Firsov, Zh. Eksp. Teor. Fiz. 43, 1843 (1962), [Sov. Phys. JETP 16, 1301 (1962)].

32 W. von der Linden, Phys. Rep. 220, 53 (1992).

${ }^{33}$ R. Blankenbecler, D. J. Scalapino, and R. L. Sugar, Phys. Rev. D 24, 2278 (1981).

34 M. Hohenadler, D. Neuber, W. von der Linden, G. Wellein, J. Loos, and H. Fehske, cond-mat/0412010 (unpublished).
35 P. E. Kornilovitch, Phys. Rev. Lett. 81, 5382 (1998).

36 P. E. Kornilovitch, Phys. Rev. B 60, 3237 (1999).

37 The relatively large statistical errors of $\Delta E$ and the resulting uncertainties in the estimate of the critical temperature are due to the addition of the absolute errors to obtain the error of the (small) binding energy.

38 A discussion of the validity of the two pictures has been given by Edwards. 